\title{
Síndrome neurológico transitorio y anestesia espinal-epidural combinada: ¿̇expresión de toxicidad por anestésico local?
}

\author{
Transient neurologic syndrome and combined spinal-epidural \\ anaesthesia: Evidence of toxicity from local anaesthetic?
}

Jorman H. Tejada', Vladimir Rivas²

\begin{abstract}
Resumen
Se reporta el caso de una paciente de 39 años de edad quien es llevada a histerectomía abdominal total, cistouretropexia retropúbica mas colporrafia posterior bajo anestesia espinal, realizada con bupivacaína hiperbárica al $0,5 \%$ y uso de analgesia peridural; al momento del retiro de catéter peridural presenta sintomatología neurológica en miembros inferiores. Se diagnostica síndrome neurológico transitorio (TNS) y se realiza seguimiento hasta la desaparición de su sintomatología. Es importante entender que a pesar de la evolución benigna de esta entidad, este cuadro es de interés, ya que su verdadero impacto clínico aún está por definirse debido a que los primeros reportes en la literatura datan del año 1993 y que su incidencia presenta gran variabilidad. En este artículo se hace una revisión de la literatura actual sobre TNS, además se describen las características principales de dicha patología.
\end{abstract}

Palabras clave: anestesia espinal, anestésicos locales, complicaciones, síntomas neurológicos transitorios, TNS.

\begin{abstract}
This article reports the case of a 39-year-old patient who underwent a local abdominal hysterectomy, Retropubic cystouretropexy as well as a posterior colporrhaphy under spinal anaesthetic, carried out with $0.5 \%$ hyperbaric bupivacaine and the use of peridural anaesthesia. When removing the peridural catheter neurological syndromes presented themselves in the lower limbs. Transient neurologic syndrome (TNS) was diagnosed and monitored until the symptoms disappeared. It is important to understand that despite the benign evolution of this syndrome these symptoms are of interest, given that their real clinical impact is yet to be defined as the first reports only date back to 1993 and its impact varies widely. This article revises the current literature about TNS and also describes the main characteristics of this condition.
\end{abstract}

Key words: spinal anesthesia, local anesthetics, complications, transient neurologic symptoms, TNS.

1. MD. Neuroanestesiólogo. Docente Coordinador Postgrado Anestesiología y Reanimación, Universidad Surcolombiana, Hospital Universitario de Neiva, Colombia.

2. MD. Residente de Anestesiología y Reanimación, Universidad Surcolombiana. Neiva, Colombia.

Correspondencia: Jorman H. Tejada. Correo electrónico: j1 tejadap@yahoo.es.

Recibido: 02/05/2015-Revisado: 22/07/2015-Aceptado: 22/11/2015 
R.F.S Revista Facultad de Salud

Julio-Diciembre de 2015;7(2): 47-50
Síndrome neurológico transitorio y anestesia espinal-epidural combinada: ¿expresión de toxicidad por anestésico local?

\section{Introducción}

La era de la anestesia regional comienza con el descubrimiento de los anestésicos locales por Köller en $1884^{1}$; en el año 1898 , Bier introduce la anestesia espinal en el mundo ${ }^{2}$, pero es Cathelin y Sicard en $1901^{3}$ los que descubren la anestesia epidural convirtiéndose en una técnica que revolucionó la practica de la anestesia. Una de las complicaciones postoperatorias del uso de la anestesia espinal descritas en la literatura es el Síndrome Neurológico Transitorio (TNS), previamente conocidos como irritación radicular transitoria; cuyos síntomas típicos son más sugestivos de patología musculo esquelética que neurológica, por lo cual algunos autores han propuesto llamarlo "síntomas musculo esqueléticos postespinales" por ser provocados posterior a la aplicación subaracnoidea de anestésico local.

La incidencia del Síndrome Neurológico Transitorio es muy variada, en 1998 Freedman realizó un trabajo multicéntrico, prospectivo, que involucró 1.863 pacientes, describiendo una incidencia de TNS de $11,9 \%$ en quienes recibieron lidocaína, $1,3 \%$ con bupivacaína y $1,6 \%$ con tetracaína; al analizar sólo el grupo que recibió lidocaína, la incidencia descrita en pacientes hospitalizados operados en decúbito supino fue de $3,1 \%$, versus $24,3 \%$ en pacientes de régimen ambulatorio y en posición de litotomía ${ }^{4,5}$. Datos que se correlacionan con el meta análisis de Eberhart ${ }^{6}$, en 2.813 pacientes con una incidencia de TNS de $19.1 \%$ en quienes recibieron mepivacaína, $16.9 \%$ con lidocaína, $1.7 \%$ con prilocaína y $1.1 \%$ con bupivacaína; y con el meta análisis de Zaric $^{7}$ en 1.347 pacientes con incidencia global de TNS de $8.7 \%$ y de $13.7 \%$ con lidocaína ${ }^{4-6}$; en 1993 Schneider y col. ${ }^{7}$, describieron formalmente el TNS al publicar los casos de cuatro pacientes que tras una anestesia espinal en posición de litotomía tuvieron dolor en espalda, glúteos y muslos con irradiación a piernas en el postoperatorio. Todos los pacientes recibieron lidocaína al 5\% y se recuperaron completamente en el postoperatorio inmediato del bloqueo espinal. El curso de estos síntomas fue auto limitado 3 a 5 días y no se perpetuaron ni se relacionaron con secuelas neurológicas.

\section{Caso clínico}

Mujer de 39 años, con diagnóstico ecográfico de miomatosis uterina y clínico de cistocele grado II-III y sobrepeso con índice de masa corporal (IMC) de 31, se decide realización de histerectomía abdominal total, cistouretropexia retropúbica más colporrafia posterior.

En la valoración preoperatoria se detecta sobrepeso y alergia a la dipirona, los paraclínicos (hemograma, bioquímica y coagulación) eran normales; en sala de cirugía se realiza monitorización básica (cardiovisoscopio, presión arterial no invasiva y pulsioximetría) se canaliza vía periférica $18 \mathrm{G}$ en miembro superior izquierdo, se posiciona en sedestación y se realiza previa asepsia y antisepsia técnica peridural a nivel L1-L2 con aguja Touhy No 16, avance cefálico de catéter peridural a $12 \mathrm{~cm}$, prueba de pérdida de resistencia negativa y test dose con $3 \mathrm{~cm}$ de lidocaína $2 \%$ con epinefrina, $60 \mathrm{mg}$ y 15 $\mu$ cg respectivamente, negativa a los 3 minutos, seguido de administración de anestesia subaracnoidea nivel L2-L3, vía medial, con aguja $26 \mathrm{G}$ Quinke, se administraron $10 \mathrm{mg}$ de bupivacaína hiperbárica (Braun Medical - Bupivacaína hiperbara $0,5 \% 10 \mathrm{~mL} \circledast$ ), morfina $100 \mu \mathrm{cg}$ y fentanil $25 \mu \mathrm{cg}$, sin síntomas secundarios ni complicaciones inmediatas durante el procedimiento. Tras la técnica anestésica se posiciona en litotomía estándar, el nivel sensitivo alcanzado a los 10 minutos de la anestesia subaracnoidea fue T7-T8. El procedimiento duró 120 minutos con estabilidad hemodinámica y ventilatoria, es llevada posteriormente a la unidad de cuidados pos anestésicos (UCPA) dónde permaneció 180 minutos hasta recuperación completa del bloqueo sensitivo y motor, posterior egreso a hospitalización ginecología.

A la 24 horas se realiza visita para retiro de catéter peridural, refiriendo aparición de dolor lumbar bilateral urente, con irradiación a glúteos y miembro inferior derecho cara anterior y posterior del muslo de manera espontanea sin asociación a traumatismos ni movimientos forzados, disminución de la fuerza muscular y alteración de la sensibilidad en el área en mención, niega cefalea o dolor en la zona de la punción lumbar, no presenta relajación de esfínteres ni fiebre.

$\mathrm{Al}$ examen físico y neurológico, afebril, área de punción sin signos de inflamación ni dolor a la palpación superficial, sin contracturas musculares para espinales bilaterales en región lumbar, se evidencia monoparesia proximal y para la flexión del pie derecho, hipoestesia en parches L1-L2-L3 derecho, L3-L4-L5 izquierdo, reflejos osteotendinosos y cutáneos abdominales conservados y sin signos de irritación meníngea; se observa mejoría en decúbito supino y con la administración de ibuprofeno. Se realiza RNM torácica y lumbosacra que evidencia imagen hipointensa definida como artificio a nivel de T9; sin compromiso de cordón medular.

Se dio de alta con manejo expectante, seguimiento ambulatorio con explicación de posible diagnóstico de TNS y el curso benigno que lo caracteriza, medidas posturales, fisioterapia y tratamiento analgésico mientras persistan los síntomas. Cerca de 21 días después del procedimiento quirúrgico presenta mejoría de la sintomatología con persistencia únicamente de hipoestesia por lo que se realizo electromiografía con reporte de músculos paraespínales lumbares de miotoma L2 derecho con signos de inestabilidad de membrana y también en aductor longus derecho con unidad motora de morfología normal; adicionalmente, se realizó neuroconducción con reporte de nervio tibial, peroneo y femoral bilateral con latencia, amplitud y velocidad de conducción normal, onda $\mathrm{F}$ de nervios tibiales con latencia y resistencia adecuadas, reflejo $\mathrm{H}$ de nervio tibial normal.

Se dan indicaciones de acudir al servicio de urgencias en caso de fiebre, aumento del dolor o agudización de alteraciones sensitivas o motoras; a los 30 días disminución de la sintomatología y al día 40 completa recuperación del cuadro descrito.

\section{Discusión}

Complicaciones como lesiones neuronales y toxicidad por anestésicos locales son comunes a todas las técnicas de 
anestesia regional. Todos los candidatos potenciales para la anestesia regional deben ser cuidadosamente evaluados e informados de las posibles complicaciones y si existe un riesgo significativo de lesiones, éstas técnicas deben evitarse. Las causas del dolor lumbar son múltiples y van desde simples lumbagos hasta dolores de origen musculo-esquelético, neurológico, psicógeno e idiopático. La paciente negaba haber sufrido traumatismo o haber realizado movimientos bruscos o posturas forzadas durante su estancia hospitalaria, carecía de antecedentes de patología lumbar o episodios de lumbalgia; sin embargo el dolor se describió como lancinante con disestesias y sensación de quemazón, se irradiaba tanto a la cara anterior como a la cara posterior del muslo; carecía de alteración de los reflejos tendinosos, y presentaba déficit motor; el dolor no se modificaba con la flexión, extensión o rotación de la columna, y no se apreciaron contracturas de la musculatura paraespinal ipsi o contralateral.

El TNS se caracteriza por dolor postoperatorio, ardor o disestesias en glúteos, extremidades inferiores, gastronemios y para algunos autores, un dolor lumbar que puede ocurrir hasta en el $50 \%$ de los pacientes $^{8}$, con completa resolución de los síntomas, sin hallazgos imagenológicas ni en la electromiografía que pudieran indicar lesiones neurológicas. Esto hace que el diagnóstico diferencial inicial con un hematoma epidural o con una lesión de las raíces nerviosas sea difícil ya que estas entidades pueden presentar síntomas similares.

La aparición de los síntomas posterior a la anestesia espinal es generalmente entre 24 a 48 horas, con una duración de 6 horas a una semana ${ }^{4}$, la literatura describe una duración promedio de 2 a 3 días ${ }^{9}$. El $90 \%$ se resuelve en una semana ${ }^{4}$, en nuestro caso la recuperación completa de la paciente se documento en el día 40.

La causa de TNS ha sido investigado en diversos estudios asociándolo con la concentración de lidocaína ${ }^{10}$, osmolaridad $^{11}$, concentración de dextrosa ${ }^{11}$, posición de litotomía $^{12}$, cirugía ambulatoria o deambulación temprana ${ }^{13}$; la mala distribución del anestésico local encontrado con el uso de agujas espinales punta de lápiz o de microcatéteres espinales ${ }^{14,15}$ pueden asociarse a lesiones neurológicas pero no a TNS. Los únicos factores que se asociaron con un incremento en el riesgo de TNS fueron la anestesia espinal con lidocaína, la posición de litotomía y la cirugía ambulatoria ${ }^{13}$. A pesar de su evolución benigna, este cuadro es de interés, ya que su verdadero impacto clínico aún está por definirse.

La incidencia reportada posee una gran variabilidad, oscilando entre un $0 \%-37 \%$, según la población estudiada ${ }^{16}$, los pacientes que son llevados a cirugía en posición de litotomía tienen una incidencia de TNS aproximada del 30 al $36 \%{ }^{17}$, artroscopia de rodilla del 18 al $22 \%$, posición supina del 4 al $8 \%{ }^{18}$, estos datos ayudarían a explicar la gran variabilidad que se reportan en los estudios.

En una revisión Cochrane ${ }^{19}$, el riesgo relativo para TNS después de anestesia espinal con lidocaína fue del 7.31 (95\% CI 4.16-12.86) comparado con otros anestésicos locales como bupivacaína, prilocaína, procaína, levobupivacaína, ropivacaína y 2 clorprocaína; encontrándose que la mepivacaína presenta resultados similares a la lidocaína.
Las posibles causas de TNS incluyen toxicidad por anestésicos locales, trauma directo con la aguja, isquemia neural secundaria al estiramiento del nervio ciático, posición del paciente, espasmo muscular, puntos gatillo miofasciales, movilización temprana o irritación del ganglio de la raíz dorsal, sin embargo la causa real del TNS sigue siendo una incógnita.

Yamashita y col. ${ }^{20}$, compararon los efectos de los anestésicos locales, sobre las concentraciones de glutamato en una muestra de líquido cefalorraquídeo (LCR), con los cambios neurológicos e histopatológicos. Con la administración de anestésico local encontraron un aumento de las concentraciones de glutamato en el LCR y cambios en las motoneuronas del cordón espinal lumbar (sugiriendo daño en las raíces dorsales y ventrales). Demostraron que la neurotoxicidad derivada de la lidocaína era la más importante, siendo la ropivacaína la menos neurotóxica y concluyeron que existe una relación entre el TNS y el daño neurológico persistente. Así mismo consideraron que el TNS representa la entidad de lesión neurológica menor de toxicidad por anestésico local de un espectro en el que la cauda equina representaría el extremo mayor.

Kasaba y col. ${ }^{21}$, intentaron determinar la toxicidad in vitro de la lidocaína, ropivacaína, mepivacaína y bupivacaína, examinando los cambios en cultivos neuronales de la Lymnaea stagnalis encontrando cambios en el crecimiento de los conos y las neuritas axonales, así como daño tisular a los 30 minutos de la administración de estos anestésicos locales. Coincidieron con los otros autores en que la lidocaína es más tóxica que la ropivacaína y la bupivacaína, por lo que conlleva más riesgo de daño tras la administración subaracnoidea.

La terapia hasta el momento incluye analgésicos antiinflamatorios no esteroideos (AINES), opioides, relajantes musculares y manejo sintomático. Dentro de los AINES más utilizados están: ibuprofeno, naproxeno y ketorolaco. Entre los relajantes musculares más usados está la ciclobenzaprina. Estos últimos disminuyen la actividad motora tónica y son de utilidad en aquellos pacientes que evolucionan con un componente muscular importante.

El paciente debe estar en posición cómoda, considerando elevación de las piernas con almohadas y calor local. Si no responde a lo anterior, se puede intentar tratamiento con opiáceos, fisioterapia, estimulación nerviosa eléctrica transcutánea e infiltración de puntos gatillo, cuando el dolor es muy severo ${ }^{8}$.

\section{Conclusiones}

El TNS se define basado en dos premisas, la aparición de sintomatología entre 24 a 48 horas posterior a la anestesia espinal y la desaparición de la sintomatología sin existencia de déficit permanente.

Los factores asociados con la aparición de esta entidad son la aplicación de lidocaína espinal, con una mayor incidencia que con los otros anestésicos locales, sin olvidar que también pueden aparecer con el uso de éstos, como en nuestro caso; posterior a anestesia espinal con bupivacaína, 
R.F.S Revista Facultad de Salud

Julio-Diciembre de 2015;7(2): 47-50
Síndrome neurológico transitorio y anestesia espinal-epidural combinada: ¿expresión de toxicidad por anestésico local? posición del paciente durante la cirugía principalmente en litotomía y un mayor número de casos reportados desde la introducción de la cirugía ambulatoria.

El TNS seguirá siendo un reto diagnóstico y una complicación hasta el momento benigna de un hallazgo relativamente reciente relacionado con la administración de anestesia espinal, requiriendo mas estudios para poder aclarar esta entidad.

\section{Referencias}

1. Köller C. The use of cocaine for producing anaesthesia of the eye [translated and reprinted]. Lancet 1884;11:9902.

2. Bier A. Experiments regarding cocainization of the spinal cord [in German]. Zietschr Chir 1899;51:361-9.

3. Cathelin F, Sicard J. Discovery of epidural anesthesia [translated and published in classical file]. Surv Anesthesiol 1979;23:271-3.

4. Freedman J, Li D, Drasner K, et al. Transient neurologic symptoms after spinal anesthesia. Anesthesiology 1998;89:633-41.

5. Eberhart L, Morin A, Kranke P, et al. Transient neurologic symptoms after spinal anesthesia. A quantitative systematic overview (meta- analysis) of randomized controlled studies. Anaesthesist 2002;51:539-46.

6. Zaric D, Christiansen C, Pace N, et al. Transient neurologic symptoms after spinal anesthesia with lidocaine versus other local anesthetics: a systematic rewiew of randomized, controlled trials. Anesth Analg 2005; 100:1811-16.

7. Schneider $M$, Ettlin T, Kaufmann $M$, Schumacher $P$, Urwyler A, Hampl $K$ et al. Transient neurologic toxicity after hyperbaric subarachnoid anesthesia with 5\% lidocaine. Anesth Analg 1993;76(5):1154-1157.

8. Pollock J. Neurotoxicity of intrathecal local anaesthestics and transient neurological symptoms. Best Practice and Reserch Clinical Anaesthesiology 2003;17:471-84.

9. Liu S, McDonald S. Current issues in spinal anesthesia. Anesthesiology 2001;94:888-906.

10. Pollock JE, Liu SS, Neal JM, Stephenson CA. Dilution of spinal lidocaine does not alter the incidence of transient neurologic symptoms. Anesthesiology 1999;90:445-50.
11. Hampl KF, Schneider MC, Ummenhofer W, Drewe J. Transient neurologic symptoms after spinal anesthesia. Anesth Analg 1995;81:1148-53.

12. Freedman JM, Li DK, Drasner K, Jaskela MC, Larsen B, Wi S. Transient neurologic symptoms after spinal anesthesia: an epidemiologic study of 1,863 patients. Anesthesiology 1998;89:633-41.

13. Lindh A, Andersson AS, Westman L. Is transient lumbar pain after spinal anaesthesia with lidocaine influenced by early mobilisation. Acta Anaesthesiol Scand 2001;45:290-3.

14. Beardsley D, Holman S, Gantt R, Robinson RA, Lindsey J, Bazaral M, Stewart SF, Stevens RA. Transient neurologic deficit after spinal anesthesia: local anesthetic maldistribution with pencil point needles? Anesth Analg 1995;81:314-20.

15. Rigler ML, Drasner K. Distribution of catheter injected local anesthetic in a model of the subarachnoid space. Anesthesiology $1991 ; 75: 684-92$

16. Pollock J. Transient neurologic symptoms: etiology, risk, factors, and management. Reg Anesth Pain Med 2002;27:581-86.

17. Hampl KF, Heinzmann-Wiedmer S, Luginbuehol I, Harms C, Seeberger M, Schneider M, Drasner K: Transient neurologic symptoms after spinal anesthe- sia. Anesthesiology 1998;88:629-633.

18. Pollock JE, Neal JM, Stephenson CA, Wiley C. Prospective study of the incidence of transient radicular irritation in patients undergoing spinal anesthesia. Anesthesiology 1996;84:1361-1367.

19. Zaric D, Pace NL. Transient neurologic symptoms (TNS) following spinal anaesthesia with lidocaine versus other local anaesthetics. Cochrane Database of Systematic Reviews 2009, Issue 2. Art. No.: CD003006. DOI: 10.1002/14651858.CD003006.pub3.

20. Yamashita A, Matsumoto M, Matsumoto $S$, et al. A comparison of the neurotoxic effects on the spinal cord of tetracaine, lidocaine, bupivacaine, and ropivacaine administered intrathecally in rabbits. Anesth Analg 2003;97:512-518.

21. Kasaba T, Onizuka S, Takasaki M. Procaine and mepivacaine have less toxicity in vitro than other clinically used local anesthetics. Anesth Analg 2003;97:85-90. 